\title{
A Case of Amyloidosis Cutis Dyschromica
}

\author{
Gu Hongzhi ${ }^{1}$, Zhang Lian ${ }^{1}$, Xiao Yan ${ }^{1, *}$ \\ ${ }^{1}$ Department of Dermatology, First Affiliated Hospital of Army Military Medical University, Chongqing, 400038, China
}

\begin{abstract}
The patient was a 56-year-old female. The whole body has brown patches for 16 years. Dermatological examination: brown patches were observed on the trunk and lower limbs, with scattered hypomigmentation patches and symmetrical distribution of skin lesions. Histopathology of the skin showed mild hyperkeratosis in the epidermis, focal liquefaction degeneration in the basal layer, masses of light red stained material in the dermal papilla, scattered or small patches of lymphocytes and tissue cell infiltration around capillaries in the superficial dermis, more pigmentophagocytes and positive methyl violet staining were observed. Diagnosis: cutaneous amyloidosis with abnormal pigmentation. The patient is still being followed up.
\end{abstract}

\section{Introduction}

The 56-year-old female patient has presented brown patches all over her body for 16 years. Sixteen years ago, brown patches appeared without obvious causes and were not taken seriously for they only made her itch occasionally. Later, the patches gradually expanded without symptoms of telangiectasia, blisters and the like. The patient had no special medical or personal history, and none of her family members suffered from similar conditions. According to the skin check, visible brown patches were found on the trunk and both lower limbs, on which there were scattered hypopigmented macules partially fused and with different diameters in the form of atrophic round white spots (Figures $1 \mathrm{a}$ and $1 \mathrm{~b}$ ). Skin lesions were symmetrically distributed mainly in the waist, abdomen and lower limbs, and were not scaly and slightly lichenoid. On the curved sides of the lower limbs appeared more inflammatory dark red spots, few of which began to scab. Face, palms and soles of the feet were not affected. The lab examinations found no abnormalities in routine blood, urine, feces, liver function and kidney tests. A chest X-ray test showed fatty liver on abdominal ultrasound. The cutaneous histopathology revealed slight epidermis hyperkeratosis, focal liquefied degeneration in the basal layer, crumby reddish matters in papillare cutis, scattered or small strips of lymphocytes and macrophages infiltration around capillary vessels in the superficial dermis, and more chromophagocytic cells (Figure 2a); the methyl violet staining results are positive (Figure $2 b$ );

Diagnosis: the patient was diagnosed with amyloidosis cutis dyschromica (ACD).
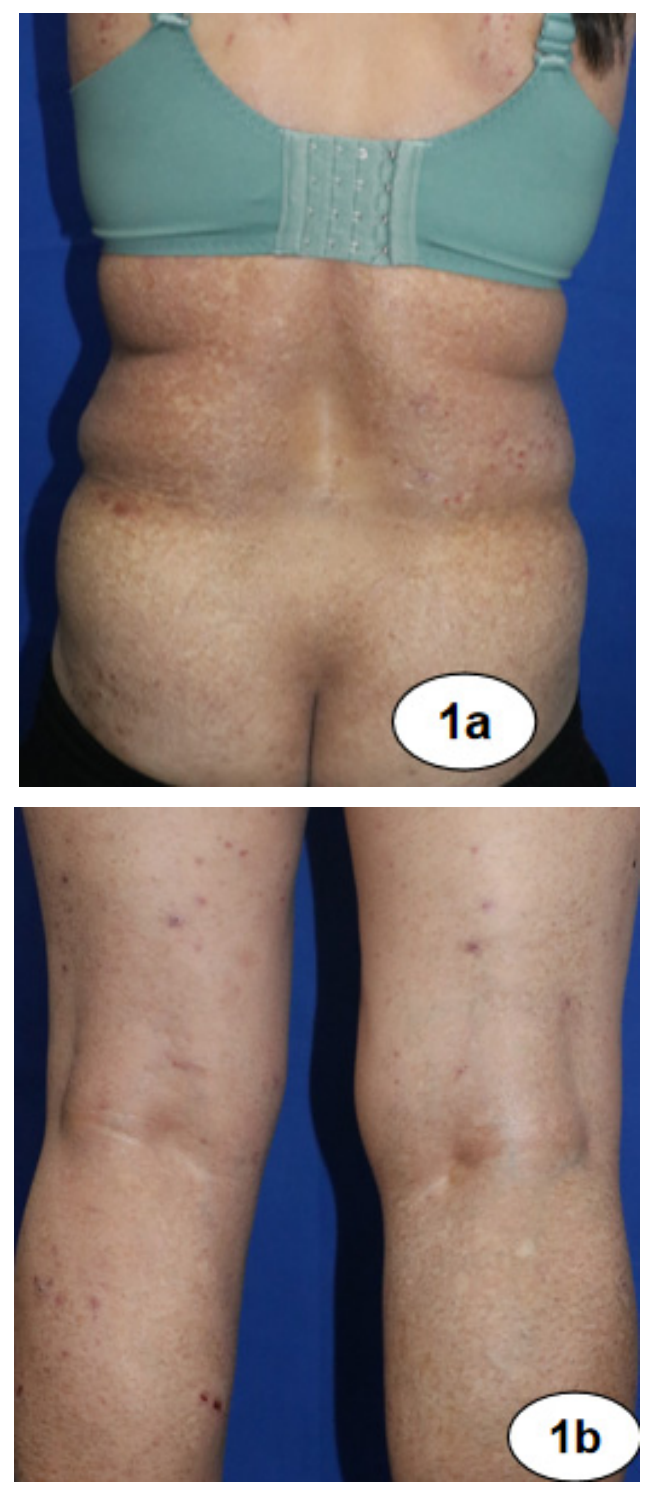

\footnotetext{
*Corresponding author: 726438380@qq.com
} 

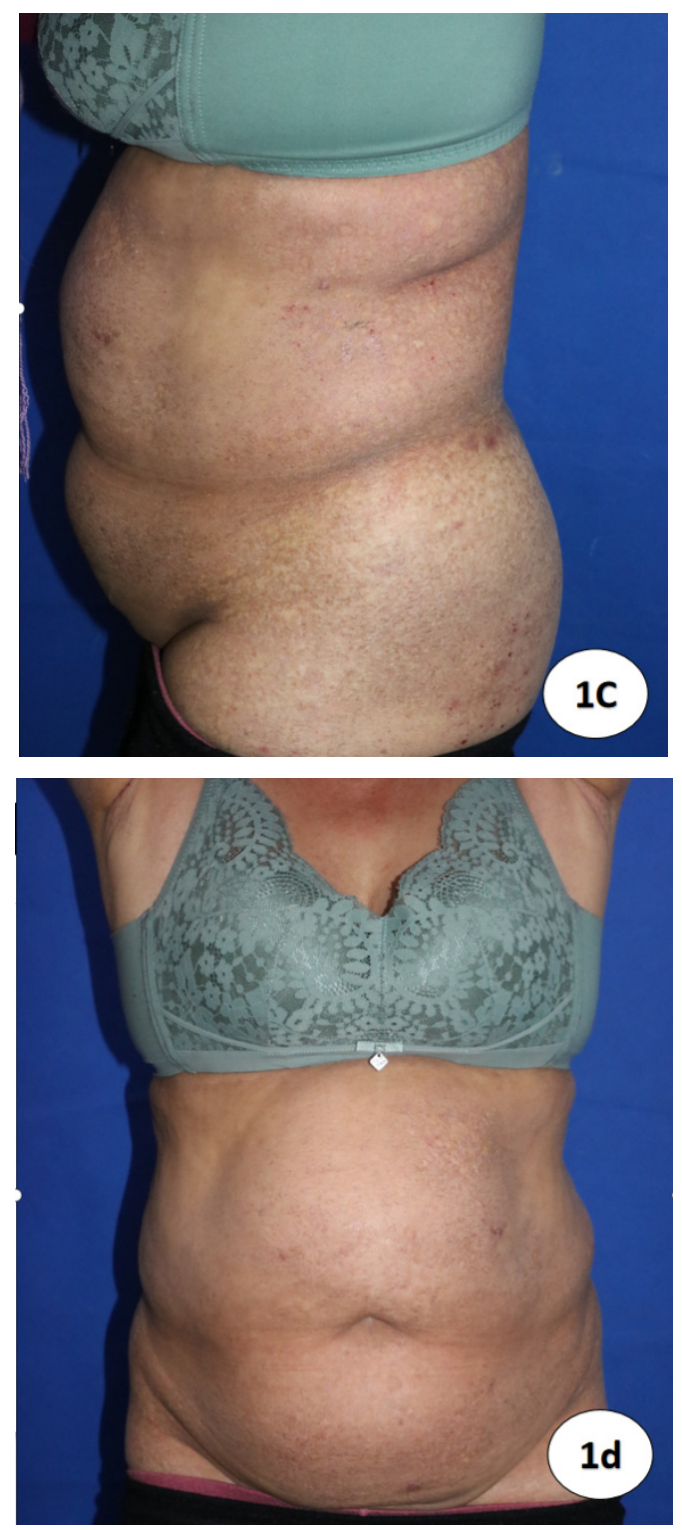

Figures 1a, 1b, 1c, 1d: visible brown patches were found on the trunk and both lower limbs, on which there were visible scattered hypopigmented macules partially fused and with different diameters in the form of atrophic round white spots.

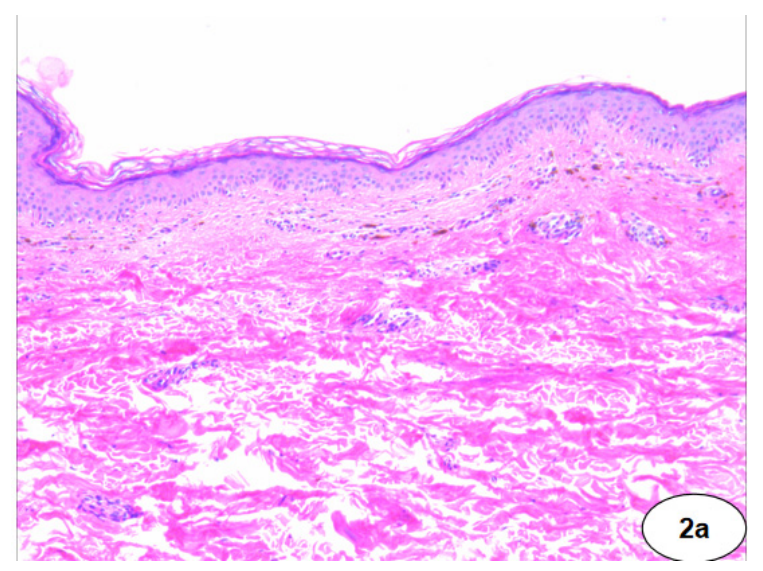

Figure 2a: slight epidermis hyperkeratosis, focal liquefied degeneration in the basal layer, crumby reddish matters in papillare cutis, scattered or small strips of lymphocytes and macrophages infiltration around capillary vessels in the superficial dermis, more chromophagocytic cells (HE,200×)

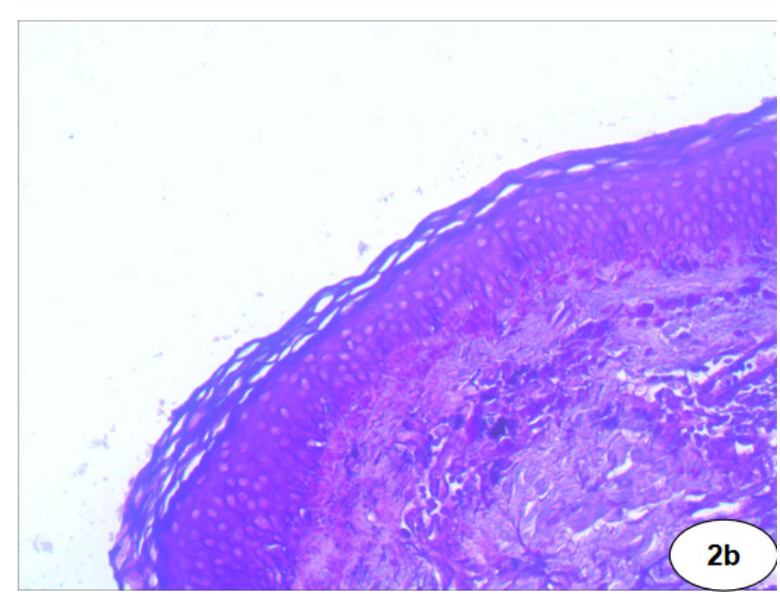

Figure 2b: positive methyl violet staining results $(200 \times)$

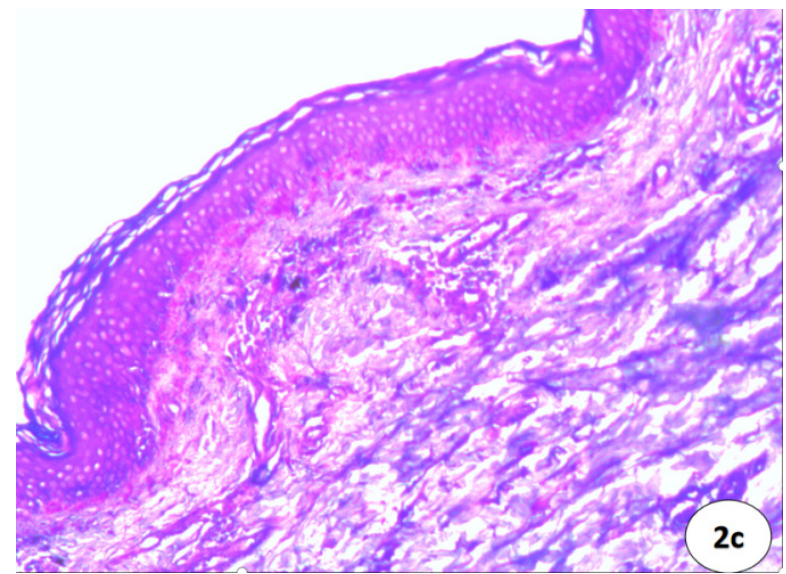

Figure 2c: (HE,200×)

\section{Discussion}

$\mathrm{ACD}$, as a rare type of primary amyloidosis, was first reported by Morishima ${ }^{[1]}$ in 1970 . Its clinical features include that ${ }^{[2]}$ : (1) It often attacks the preadolescents, some of whom have family history; (2) Most patients are Asians, such as Chinese, Japanese and Thai people; (3) Skin lesions show reticulate acropigmentation accompanied by spotted hypopigmentation; (4) There are no obvious subjective symptoms, such as itch or occasionally slight itch; (5) Pigment changes almost affect the skin of the whole body, but the skin of the face, neck and hands are lighter than the non-exposed parts. The mucous membranes, palms and plantars, hair, nails and teeth are not involved, and there is no system abnormality. (6) the disease develops slowly, and usually has no erythema, papules, blisters, adermotrophia, angiotelectasis and other symptoms. The principal manifestations of the disease include: amyloid deposition in dermal papilla, and positive methyl violet staining or Congo red staining results. The patient presents obvious brown pigmentation accompanied by hypopigmented patches. Skin lesions are obviously found on the trunk and limbs, and not obviously on hands or feet. There are no discomforts such as itching, and methyl violet staining results are positive. Those manifestations are in line with clinical features of this disease, except the disease time 
that the patient forgets. This disease should be differentiated from the poikilo-dermalike cutaneous amyloidosis (PCA syndrome for short), which is very similar to ACD in tissue biopsy and clinical features some of which overlap with each other ${ }^{[3]}$. Clinical features of the PCA syndrome mainly include ${ }^{[4]}$ : (1) Adermotrophia, hyperpigmentation, hypopigmentation, angiotelectasis, vesiculation, palmoplantar keratosis and others (2) lichenoid papules; (3) Being sensitive to light; (4) That histopathological examination shows amyloid depositions in the dermal papilla; And (5) short stature. This patient has no lichenoid papules, blisters, palmoplantar keratosis, photosensitivity, and short stature. Therefore, the diagnosis of PCA can be ruled out, which is consistent with the diagnosis of dyspigmented skin amyloidosis. There are also literatures ${ }^{[3]}$ reporting the appearance of blisters in ACD. Therefore, these two kinds of skin amyloidosis are very easy to be confused in clinical practice, and they are easy to be mistaken for rash. This requires the doctor to ask the patient's medical history and examine the body carefully. This disease needs to be differentiated from the following diseases: (1) Idiopathic punctate leukoplakia: The rash is the size of a millet to the size of a soybean, which is mostly affected by people over 50 years old; (2) Pan-type vitiligo: The color of the skin lesions varies depending on the degree of depigmentation, and can be introverted The outer appearance is white, gray, and nearly normal skin color, and the peripheral pigment is darkened. Pathologically, the epidermal melanin is reduced or absent; (3) Civatte skin heterochromia: It occurs in the "v"-shaped area of middle-aged women, and the typical skin lesions are symmetrical. Red-brown reticulum pigmentation with telangiectasia, and epidermal atrophy at the late stage; (4) Glossy lichen: It is common in adolescent males, miliary-sized round flat bright papules, skin color or pale white, pathology sees isolated focal lymphocytes in the dermis It is infiltrated with tissue cells, and the epidermal protrusions on both sides are elongated in a "spheroidal shape". And distinguish it from hereditary generalized pigmentary dysplasia, congenital pigmentary dysplasia, xeroderma pigmentosum, and generalized reticular pigmented skin disease, etc. Besides, ACD should also be differentiated from dyschromatosis universalis hereditaria, congenital dyschromatosis, xeroderma pigmentosum, generalized dermatopathia pigmentosa reticularis and the like, and histopathological examination will help to do so. At present, the pathogenesis of dyspigmented skin amyloidosis is still unclear. Some patients develop onset before puberty and have family inheritance ${ }^{[5]}$, suggesting that inheritance may play a role. Recently, Fernandes et al. ${ }^{[6]}$ found that the amyloid in dyspigmented skin amyloidosis lesions may be derived from keratin, because these amyloids are positive when stained with keratin. Yang et al. ${ }^{[7]}$ found that the level of glycoprotein non-metastatic melanoma $b$ in patients with dyspigmented skin amyloidosis decreased significantly and related genes were also mutated. It is speculated that this may be one of the causes of dyspigmented skin amyloidosis, because the protein has multiple functions: it participates in the apoptosis of keratinocytes and other cells, and affects the development and adhesion process of melanocytes. It is speculated that non-metastatic melanin Mutations in the oncoglycoprotein gene cause abnormal apoptosis of keratinocytes in the skin lesions and abnormal function of melanocytes, which leads to amyloid deposition and changes in skin color. The disease may also be related to skin and epidermal damage caused by ultraviolet radiation and friction. Although the patient only showed changes in skin pigmentation and there was no obvious system damage. However, the damage area is large and may gradually spread, which will greatly affect the patient's appearance, bring great inconvenience to life and work, and easily cause psychological burden. At present, various treatment methods for the treatment of skin amyloidosis can be applied, but there is no effective and recognized treatment method. The patients are recommended not to get too much sun exposure and to make use of sun creams and moisturizers. It is also reported in relevant literature ${ }^{[8]}$ that taking Vitamin A Acid drugs orally can better help cure the disease. In addition, external use of keratolytics, glucocorticoid, capsaicin, methyl-carbonate sulfoxide and laser is also recommended. The treatment time of this patient was short, a clinical follow-up of the patient is still being conducted currently.

\section{Conclusion}

Dyspigmented skin amyloidosis is rare in clinical practice ${ }^{[2]}$. Therefore, in clinical cases, if the onset occurs before puberty, the first onset is in the calf, and the clinical manifestations are patients with diffuse reticular pigmentation with punctate hypopigmentation spots, which need to be alert to the occurrence of this disease.

\section{Reference}

1. Morishima T. A clinical variety of localized cutaneous amy- loidosis characterized by dyschromia (amyloidosis cutis dys- chromica) [J]. Jpn J Dermatol Series B, 1970, 1:80.

2. Qiao J, Fang H, Yao H. Amyloidosis cutis dyschromica [J]. Orp J R are Dis, 2012, 7:95.

3. Pei Xiaoping, Wang Yu, Liu Hongfang, Xue Ruzeng, Pan Huiqing, Lin Eryi, Gu Mei. Clinical Analysis of 6 Cases of Amyloidosis Cutis Dyschromica [J]. Journal of Clinical Dermatology, 2017, 46 (09): 633-635.

4. Xie Jie, Lin Eryi, Ai Jing, Liu Yahui, Que Shouhong, Li Ming, Yang Bin, Gu Youshou. A Case of Amyloidosis Cutis Dyschromica [J]. Journal of Diagnosis and Therapy on Dermato-venereology, 2016, 23 (03): 188-189.

5. Hermawan M, Rihatmadja R, Sirait SP. Familial amyloidosis cutis dyschromica in three siblings: report from indonesia [J]. Dermatol Reports, 2014, 6(1): 5375 . 
6. Fernandes NF, Mercer SE, Kleinerman R, et al. Amyloidosis cutis dyschromica associated with atypical Parkinsonism, spastic - ity and motor weakness in a Pakistani female [J]. Cutan Pathol, 2011, 38(10): 827-831.

7. Yang CF, Lin SP, Chiang CP, et al. Loss of GPNMB Causes Autosomal -Recessive Amyloidosis Cutis Dyschromica in $\mathrm{Hu}-$ mans [J]. Am J Hum Genet, 2018, 102(2): 219-232.

8. Xu Xinzhi, Wu Jie, Yang Ji, Li Ming. A Family Report of Amyloidosis Cutis Dyschromica [J]. China Journal of Leprosy and Skin Diseases, 2021, 37 (01): $40-42$

9. Chiu, F. P. C., Wessagowit, V., Cakmak, M. F., Doolan, B. J., Kootiratrakarn, T., Chaowalit, P., ... \& Onoufriadis, A. Molecular basis and inheritance patterns of amyloidosis cutis dyschromica [J]. Clinical and experimental dermatology, 2020, 45(5), 650-653.

10. Vijaikumar, M., \& Thappa, D. M. Amyloidosis cutis dyschromica in two siblings [J]. Clinical and experimental dermatology, 2001, 26(8), 674-676.

11. Garg, T., Chander, R., Jabeen, M., Barara, M., Mittal, K., Jain, M., \& Puri, V. Amyloidosis cutis dyschromica: a rare pigmentary disorder [J]. Journal of cutaneous pathology, 2011, 38(10), 823-826.

12. Madarasingha, N. P., Satgurunathan, K., \& De Silva, M. V. C. A rare type of primary cutaneous amyloidosis: amyloidosis cutis dyschromica [J]. International journal of dermatology, 2010, 49(12), 1416-1418.

13. Mahon, C., Oliver, F., Purvis, D., \& Agnew, K. Amyloidosis cutis dyschromica in two siblings and review of the epidemiology, clinical features and management in 48 casesr [J]. Australasian Journal of Dermatology, 2016, 57(4), 307-311.

14. Morales Callaghan, A. M., Vila, J. B., Fraile, H. A., Romero, A. M., \& Garcia, G. M. Amyloidosis cutis dyschromica in a patient with generalized morphoea [J]. British Journal of Dermatology, 2004, 150(3), 616-617.

15. Yang, W., Lin, Y., Yang, J., \& Lin, W. Amyloidosis cutis dyschromica in two female siblings: cases report [J]. BMC dermatology, 2011, 11(1), 1-4.

16. Kuseyri, O., Haas, D., Lang, N., Schäkel, K., \& Bettendorf, M. Amyloidosis cutis dyschromica, a rare cause of hyperpigmentation: a new case and literature review [J]. Pediatrics, 2017, 139(5).

17. Eng, A. M., Cogan, L., Gunnar, R. M., \& Blekys. Familial generalized dyschromic amyloidosis cutis [J]. Journal of cutaneous pathology, 1976, 3(2), 102-108.

18. Wang, L., Jiang, X., Zhang, N., Liu, L., Zhou, H., \& Liu, H. J. Case of amyloidosis cutis dyschromica with dermoscopy $[\mathrm{J}]$. The Journal of dermatology, 2019, 46(2), e77-e79.

19. Kutlu, Ö., Çetinkaya, P. Ö., Ünverdi, H., Vahapoğlu, G., \& Ekşioğlu, M. Late-onset amyloidosis cutis dyschromica: an unusual case [J]. Dermatology online journal, 2019, 25(4). 